\title{
Light on genome function
}

Optogenetic tools enable light-mediated control over transcription and epigenetic states in specific endogenous loci of the mammalian genome.

Studying the roles of specific genes or gene networks in cells is fundamental to biology research. Gene expression is dynamic and tightly regulated; to understand it, one needs approaches that enable fine control of the process. Recently, a variety of microbeor plant-derived light-sensitive proteins have been engineered that allow precise modulation of biochemical and electrical signaling pathways in cells by 'optogenetics'. Optogenetic tools have also been built that allow control of transgene expression with the ease of a light switch, but up until now, it had not been possible to use light to turn on or off the expression of endogenous genes or manipulate the epigenetic regulatory landscape in the genome.

Feng Zhang, who runs a laboratory at the Broad Institute of Massachusetts Institute of Technology and Harvard, was in a unique position to tackle this technological challenge. Zhang previously worked in the lab of Karl Deisseroth at Stanford University and used light-sensitive proteins from green algae and other microbes to develop a toolbox for controlling the activity of neurons in living organisms with light. Now with his own lab, he sought to expand optogenetics to the realm of genome engineering.

Zhang and his colleagues developed a new set of tools called 'LITEs', for light-inducible transcriptional effectors. The LITE system is composed of two parts: one part serves as an anchor that binds a specific place in the genome upstream of a gene one wishes to control, and the other part contains an 'effector' molecule that has the capacity to control whether gene transcription turns on or not. Critically, the two components come together only when illuminated with certain wavelengths of light.

LITE uses transcriptional activator-like effectors (TALEs) as DNA anchors fused to the light-sensitive domain Cryptochrome 2 (CRY-2). The second component includes CRY-2's interacting partner, CIB1, fused to an effector protein. Upon illumination with blue light, CIB1 binds CRY-2 at the genomic locus where CRY2-TALE is bound, allowing the effector protein to then exert positive or negative control over the gene.

Zhang's team designed LITEs that activate gene transcription in several different genomic loci. They could augment the levels of certain target genes by tenfold within just a few hours of turning a blue light on. Controlling the light intensity and illumination cycle, they could define the level of gene expression desired and ensure that the cells were healthy and happy under the spotlight.

One way in which gene expression can be turned off is by introducing epigenetic modifications at the gene's genomic locus. Based on this idea, Zhang and his team engineered LITE-based systems in which they fused repressive histone effectors, such as histone deacetylases, to the CRY-2 protein and could repress gene transcription. The approach, called 'epiLITE', opens the possibility of testing the role that epigenetic modifications have in gene regulation, something that has been hard to do with existing methods.

Researchers in Zhang's lab are primarily interested in using these tools to understand brain physiology and pathology. In this initial work, they showed that LITEs can activate the expression of specific genes in the brain of freely behaving mice. The possibility of controlling gene expression programs in living animals while they are engaged in specific behaviors enables all sorts of interesting experiments. Zhang would like to start by studying the genes involved in learning and memory. "We would like to alter the expression profile of genes involved in changing synaptic plasticity, to find out which ones are important for memory consolidation and learning," he says. Eventually, this may lead to ways in which researchers can "write new

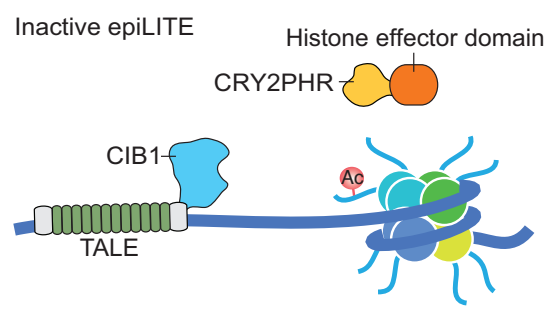

Active epiLITE

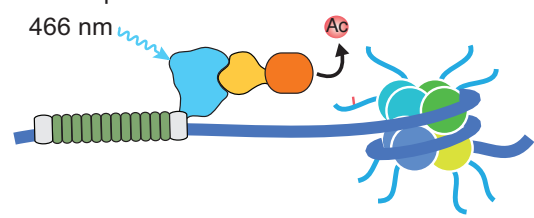

LITE-mediated epigenetic modifications. Image courtesy of the Zhang lab members.

memories" as Zhang puts it, by controlling specific genetic programs.

After a lot of hard work spent optimizing the system, Zhang and his team are now eager to get to work on those experiments. In the initial version of LITEs, the team observed relatively high basal induction of gene expression even in the absence of light and had to painstakingly go through many rounds of protein engineering to optimize the system and avoid these unwanted effects. Zhang acknowledges that this was all thanks to the efforts of Silvana Konermann and Mark Brigham, the two first authors of the paper, who are Zhang's first graduate students.

The team has also started to think of other methods for DNA targeting. Zhang is particularly interested in using RNA-guided nucleolytically inactive mutants of Cas9 instead of TALEs. "Cas9 has much higher ability to be used to target multiple loci at once," he points out, "so we envision being able to activate networks of genes rather than a single gene in the near future."

\section{Erika Pastrana}

\section{RESEARCH PAPERS}

Konermann, S. et al. Optical control of mammalian endogenous transcription and epigenetic states. Nature doi:10.1038/nature12466 (23 July 2013). 